\title{
Unrecognized Tuberculosis in a Nursing Home Causing Death with Spread of Tuberculosis to the Community
}

\author{
Kashef Ijaz, MD, MPH, "† Jennifer A. Dillaha, MD, $\neq$ Zhenhua Yang, MD, PhD,, \\ M. Donald Cave, PhD, ll and Joseph H. Bates, $M D^{*+\$}$
}

OBJECTIVES: To determine the reason for an increase in tuberculin skin test (TST) conversion in employees in a nursing home and to determine the source case responsible for spread of tuberculosis (TB) in two nursing homes and a hospital in a rural part of Arkansas using molecular and traditional epidemiological methods.

DESIGN: TB contact investigation of residents and employees of two nursing homes and a hospital.

SETTING: Two nursing homes and a hospital in rural part of Arkansas.

PARTICIPANTS: One hundred fifty-seven employees and 117 residents of two nursing homes and 211 employees of a hospital in rural part of Arkansas

MEASUREMENTS: Tuberculin skin test.

RESULTS: Analysis of room and work assignments of residents and employees who converted their TSTs in Nursing Home A showed that residents and employees in the same wing as the suspect source case were significantly more likely to have converted their TST than residents and employees in other wings $(P=.01)$. A nurse from the local hospital where the suspected source case had been sent developed a tuberculous cervical abscess, and one employee in Nursing Home A developed pulmonary TB. A visitor to Nursing Home A was diagnosed with culture-positive pulmonary TB 2 years later. Genotyping of the Mycobacterium tuberculosis isolates from the four secondary cases showed identical patterns.

CONCLUSION: Molecular and traditional epidemiological studies revealed an outbreak of TB that began in a

From the *Division of Tuberculosis and ${ }^{+D}$ Departments of Internal Medicine, ${ }^{\ddagger}$ Geriatrics, ${ }^{\$}$ Microbiology and Immunology, and $"$ Anatomy, Arkansas Department of Health, University of Arkansas for Medical Sciences, Little Rock, Arkansas; "School of Public Health, University of Michigan, Ann Arbor, Michigan.

Support was received from the Arkansas-Centers for Disease Control and Prevention Tuberculosis cooperative agreement and Centers for Disease Control and Prevention-Veterans Affairs interagency agreement for genotyping.

Address correspondence to Joseph H. Bates, MD, Deputy Health Officer, Mailstop: 45; Arkansas Department of Health, 4815 W. Markham, Little Rock, AR 72205. E-mail: jbates@healthyarkansas.com nursing home and spread to a second nursing home, a local hospital, and the community. J Am Geriatr Soc 50: 1213-1218, 2002.

Key words: tuberculosis; epidemiology; nursing home; genotyping

$I^{n}$ $n$ the United States, the incidence of tuberculosis (TB) is higher in those aged 65 and older than in any other population group except persons with human immunodeficiency virus (HIV) infection. ${ }^{1}$ The geriatric population has four times the risk of TB as that of the general population. Nursing home residence doubles the age-adjusted risk of developing TB. ${ }^{2}$

Tuberculosis that develops after entry to a nursing home may be difficult to recognize. Chronic cough, weight loss, and debility in older persons have multiple causes, which, when combined with other comorbid conditions, may confuse the clinical manifestations of TB. Undiagnosed TB in nursing home residents resulting in unrecognized transmission of Mycobacterium tuberculosis (MTB) to other residents, employees, and visitors has been well documented. ${ }^{3-6}$ Estimating the number of persons who are at increased risk of exposure to TB by virtue of employment is difficult, because the number of persons employed in the nursing home industry is unknown. In the United States, there are approximately 16,700 nursing homes housing 1.6 million residents. ${ }^{7}$ There are an estimated 75 full-time equivalent employees per 100 nursing home residents. ${ }^{7}$ The employee turnover rate in the nursing home industry is high, perhaps as high as $68 \%$ per year (John Ferguson, RN, Beverly Enterprises, personal communication, December 8, 2000). Thus, in a given year in the United States, we estimate that 2 million persons are employed in nursing homes; the actual number of persons may be higher, because many are employed on a part-time basis. These employees represent a population at increased risk of $\mathrm{TB}$ infection. The $\mathrm{TB}$ case rate for nursing home employees is three times higher than the rate expected for other employed adults of similar age, race, and sex. ${ }^{2}$

The Tuberculosis Control Program at the Arkansas Department of Health has maintained careful surveillance for TB in nursing home residents and employees in the 
state for 20 years. We recently discovered an outbreak of $\mathrm{TB}$ resulting from an unrecognized, highly infectious case that transmitted infection to 40 nursing home employees, 24 nursing home residents, two visitors, and 14 employees of a nearby hospital. Four cases of active TB resulted. We wish to describe this outbreak and the methods used to follow its spread.

\section{MATERIALS AND METHODS}

\section{Tuberculin Skin Testing}

All persons admitted to Arkansas nursing homes are screened for TB on admission. If they have a documented positive tuberculin skin test (TST), a chest $\mathrm{x}$-ray and clinical evaluation are performed. All others have a TST using a two-step method with five tuberculin units of purified protein derivative (PPD). Two-step testing is performed to reduce the likelihood that a boosted reaction will be misinterpreted as a recent infection. If the first skin test shows less than $15 \mathrm{~mm}$ induration, a second test is performed within 2 weeks. The reading of the second test is recorded as the baseline value. All persons having a reaction of $10 \mathrm{~mm}$ or greater on the second test are considered positive. Each resident with a positive TST receives a clinical evaluation that includes a chest $\mathrm{x}$-ray. Employees are also screened using the two-step TST. All employees who are TST negative are screened annually, with a TST administered on the anniversary of their employment.

\section{Culture for Mycobacterium Tuberculosis}

When a nursing home patient develops a cough that persists for 3 weeks or longer, staff are encouraged to obtain sputum for MTB culture.

\section{Genotyping of Mycobacterium Tuberculosis}

Since January 1992, the Arkansas Tuberculosis Control Program has collected and stored all available isolates from newly diagnosed cases of TB. At least one isolate from each case is subjected to IS6110-based restriction fragment length polymorphism analysis. ${ }^{8}$ A genotype database consisting of fingerprint patterns of approximately 1,514 individual Arkansas isolates has been established.

\section{RESULTS}

\section{Investigation of Outbreak in Nursing Home}

The investigation began in June 1998, when the supervisor of a 116-bed nursing home (Nursing Home A) with 127 employees noted that six of 18 employees undergoing their annual TSTs that month had become positive, a marked increase from the expected rate of less than $1 \%$. This finding prompted an evaluation of all remaining employees and all the nursing home residents to search for an undiagnosed source case as shown in Table 1. Each employee or resident with a TST conversion received a clinical evaluation and a chest $\mathrm{x}$-ray. One employee and one resident were found to have minimal chest $\mathrm{x}$-ray abnormalities. Sputum samples from each reactor were evaluated by microscopy for acid-fast bacilli (AFB) and cultured for MTB. Sputum from the resident was negative on smear and culture for AFB, but sputum from the employee (Case 1) grew MTB, although the stained smear was negative. Case 1 was a 37 -year-old woman who had worked at the nursing home for 6 years. Having only a few organisms in her sputum as judged by the negative sputum smears, she was not thought to be highly infectious. She was, therefore, an unlikely source case for the outbreak.

All employees with previously positive TSTs received clinical evaluations and chest $\mathrm{x}$-rays and all were normal. None of the residents had symptoms suggestive of TB. Four residents had abnormal chest x-rays, but none were suspicious for $\mathrm{TB}$, and their sputum cultures were negative for MTB.

Because the initial screen of employees and residents failed to identify a likely source case, "cough rounds" were made in the nursing home to collect sputum from all residents and employees who complained of a cough. This effort produced 120 sputum specimens from 41 employees and 48 residents; all were culture-negative for MTB.

In addition, a standardized epidemiological questionnaire was completed for each resident and employee to obtain demographic information and data on room assignments for residents and work assignments for staff. Through this process we learned that some employees also worked in a second nearby nursing home. The second nursing home (Nursing Home B) was a 28-bed facility with $30 \mathrm{em}$ ployees owned by the same person who owned Nursing Home A. Nursing home staff and residents often moved between these two facilities.

All current employees and residents at Nursing Home B were screened for TB, with the results shown in Table 2. The chest $\mathrm{x}$-ray of the one resident who converted her TST showed an upper lobe infiltrate of minimal extent without cavitation. A sputum-smear was negative for AFB, but positive for MTB by a nucleic acid amplification test and by culture. This resident (Case 2) was a 75-year-old woman who had been admitted to Nursing Home A in February 1998 and transferred to Nursing Home B 2 months later. The genotype of Case 2 was found to match that of Case 1.

Table 1. Tuberculosis Contact Investigation in Nursing Home A

\begin{tabular}{|c|c|c|c|c|c|}
\hline \multirow[b]{2}{*}{ Classification } & \multirow{2}{*}{$\begin{array}{c}\text { Total } \\
\mathrm{n}\end{array}$} & Previous TST + & TST - & TST Conversions & Tuberculosis Cases \\
\hline & & \multicolumn{4}{|c|}{$n(\%)$} \\
\hline Employee & 127 & $11(9)$ & $116(91)$ & $33(28)$ & $1(1)$ \\
\hline Resident & 98 & $17(17)$ & $81(83)$ & $23(28)$ & $1(1)$ \\
\hline
\end{tabular}

TST $=$ tuberculin skin test $+=$ positive; $-=$ negative. 
Table 2. Tuberculosis Contact Investigation in Nursing Home B

\begin{tabular}{|c|c|c|c|c|c|}
\hline Classification & $\mathrm{n}$ & \multicolumn{4}{|c|}{ n (\%) } \\
\hline Resident & 19 & $2(11)$ & 17 (89) & $1(6)$ & 1 \\
\hline
\end{tabular}

TST $=$ tuberculin skin test $;+=$ positive $;-=$ negative.

Because Case 2 had negative sputum smears, she was also judged not to be highly infectious and was, therefore, an unlikely source case. The search for a source case was extended to former nursing home employees and residents. During the preceding 12 months, 47 employees had left employment at either nursing home. Thirty-four were located and evaluated. None had evidence of TB, but one had a TST conversion. Twenty-two visitors to Nursing Home A requested tuberculin skin testing. One was positive, but her chest $\mathrm{x}$-ray was normal.

To search for a source case among former nursing home residents, records were reviewed for all residents who had died during the 6-month period before the outbreak was discovered. During this time, seven deaths occurred in Nursing Home A and none in Nursing Home B. Three of the seven died at the nursing home without referral to a hospital and none of these three had been diagnosed with pneumonia or a febrile illness. Of the remaining four, all had been transferred to the local hospital and had chest x-rays taken there. Review of these x-rays identified one resident (Case 3), who had a large cavitary lesion in the right upper lobe highly suspicious for TB. Case 3 was a 91-year-old woman, who was admitted to Nursing Home A in May 1995 in relatively good health. On admission, she had no pulmonary complaint, was ambulatory, had a negative TST, and had never been admitted to any other nursing home. About 2 years after admission, she developed a productive cough and became too weak to walk. Over the following 8 months she was sent to the local hospital six times, where she was treated for pneumonia on each occasion before returning to Nursing Home A. At first she had a small, right upper lobe infiltrate on chest $\mathrm{x}$-ray, but it progressed over several months to show cavitation despite treatment with antibiotics. Tuberculosis was never considered as a possible diagnosis and no sputum studies for MTB were performed. She died in June 1998, 3 years after entering the nursing home and 8 months after onset of cough. An autopsy was not performed.

It appeared that Case 3 might be the source case, but because she died several weeks before we began our investigation, alternate approaches to confirm or exclude her as the source case were required. To determine whether epidemiological data would support the possibility that Case 3 was the source case, the layout of Nursing Home A was evaluated with regard to her room assignment and the room assignments of the residents who had recently converted their TSTs. The floor plan and bed assignments in Nursing Home A are depicted in Figure 1.

Case 3 lived in the "old" wing with 29 other residents, 22 of whom were TST negative when admitted. Eleven of the 22 residents converted their TSTs. In addition, Case 2 lived in the "old" wing for 2 months during the period when Case 3 had cavitary lesions. She later developed TB, after transfer to Nursing Home B.

During the first few months of Case 3's illness, she remained mobile and continued to visit the common room where she had contact with residents living in other wings. In the north wing, eight of 28 residents had TST conversions. In the east wing, four of 22 residents also converted. On chi-square analysis, the relative risk of TST conversion for those living in the old wing with Case 3 compared with those living in the north and east wings was $2.2(P=.01)$. These data supported our suspicion that Case 3 was the source case.

The work assignments of the employees were reviewed to determine any history of contact with Case 3. Of the 33 TST conversions among employees, only 12 were assigned to work in a designated wing exclusively. Five of the 12 converters were assigned to the old wing, with the rest divided between the other two wings.

The heating and cooling system of Nursing Home A was investigated using tracer smoke studies to determine the dissemination pattern of MTB via room air circulation. It was found that the air pressure in each room was positive with respect to the hallway. In each wing, there was a centrally located return air vent in the hallway that returned air to a heating and cooling unit for recirculation. Each of the three wings had separate air handling systems that did not mix return air with that from other wings. The return air vent in the old wing was located immediately outside the door of Case 3's room.

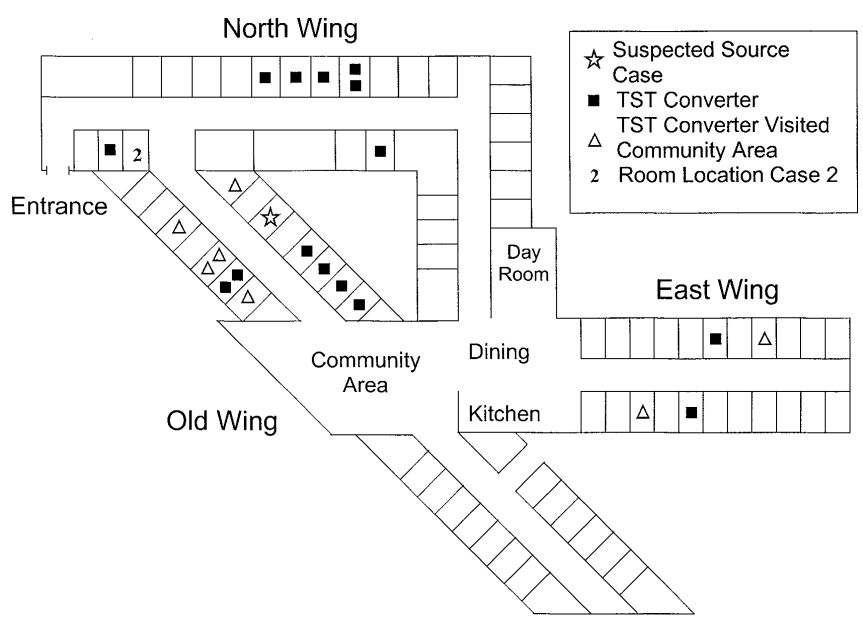

Figure 1. Room location of tuberculosis skin test (TST) converters among residents in Nursing Home A. 


\section{Contact Investigation at the Local Hospital}

To test the hypothesis that Case 3 was the source case further, the staff at the local hospital was evaluated. In this 49-bed facility, all adult medical patients are housed on a single 26-bed unit. Case 3 was admitted to this unit on all four occasions and was treated in the emergency room on two occasions. Of the 300 hospital employees, 178 had possible contact with Case 3. All were screened for tuberculosis; the results are shown in Table 3. Employees who converted their TSTs had normal chest $\mathrm{x}$-rays.

To evaluate whether the 14 TST conversions in hospital employees were due to exposure to Case 3 or to another TB case, a review of all discharges from this hospital for the period January 1, 1997, through September 30, 1998, was performed. These data were cross-matched with the state Tuberculosis Information Management System (TIMS). During this period, there were six patients with a diagnosis of TB discharged from the local hospital; four were culture-positive, one was diagnosed on clinical criteria only, and one was subsequently found to have infection with Mycobacterium avium-intracellulare.

Only one of the culture-positive cases was smear-positive, and this patient had been on TB treatment for 4 days before hospital admission. The patient was placed in an isolation room, and none of the 41 employees exposed to him had a TST conversion. Of the remaining three smearnegative, culture-positive cases, one was on TB treatment before hospitalization and was kept in isolation. The second was considered a TB suspect and was in isolation, with negative smears and cultures before being referred and diagnosed with TB at another hospital, and the third was admitted 6 months before the diagnosis of TB and had a normal chest $\mathrm{x}$-ray at the time of admission. We concluded that it is unlikely that any of these six patients transmitted infection to hospital employees.

Although Case 3 was the suspected source case, she was apparently infected after she entered the nursing home, because she was TST negative on admission in 1995. To search for a possible source for her infection, death certificates of all residents who had died in Nursing Home A and $\mathrm{B}$ during the 3-year period when Case 3 lived in Nursing Home A were reviewed. During this period, a TB case was discovered in a resident admitted to Nursing Home A in October 1995. His infecting strain of MTB showed a genotype substantially different from that harbored by Case 1 and 2, indicating that he was not the source for Case 3.

A review of the deaths at both nursing homes indicated that 88 residents at Nursing Home A and 33 resi-

Table 3. Tuberculosis Contact Investigation in the Local Hospital

\begin{tabular}{lccc}
\hline Classification & $\begin{array}{c}\text { Total } \\
\mathrm{n}\end{array}$ & $\begin{array}{c}\text { TST Conversions } \\
\mathrm{n}(\%)\end{array}$ & $\begin{array}{c}\text { Tuberculosis Cases } \\
\mathrm{n}\end{array}$ \\
\hline Exposed & 178 & $14(8)$ & 1 \\
Not exposed & 33 & $1(3)$ & 0 \\
\hline
\end{tabular}

TST $=$ tuberculin skin test. dents at Nursing Home B died during the study period. Death certificates showed that 57 of the 88 deaths at Nursing Home A were associated with pneumonia or other pulmonary conditions such as lung cancer or did not have a specific cause of death listed. Twenty-eight of these 57 died after admission to the local hospital; their chest $\mathrm{x}$-rays were reviewed. Of the 33 deaths from Nursing Home B, seven were from pulmonary conditions and were admitted to the local hospital. All together, 15 of the 35 residents who were hospitalized before death had chest $\mathrm{x}$-ray findings that could be interpreted as suspicious for $\mathrm{TB}$, but none showed cavitation or other findings highly suspicious for TB. One patient had a radiographic gibbus formation indicating possible Pott's disease, but his sputum samples were consistently smear- and culture-negative for MTB. None of the residents had HIV infection. Thus a source of infection for Case 3 was not found.

\section{Discovery of Secondary Cases of Active Tuberculosis}

After the nursing home investigation had been completed, additional patients with TB who had epidemiological links to Nursing Home A and the local hospital were discovered. The first such patient was a 31-year-old woman (Case 4) who was evaluated in another city for neck pain and was found to have cervical osteomyelitis with an associated paravertebral abscess. Surgical drainage and repair revealed the lesion to be granulomatous, and culture was positive for MTB. Case 4 worked as a nurse at the local hospital, where Case 3 had been hospitalized. During the contact investigation at the hospital, Case 4 was found to have converted her TST. Initial clinical evaluation with chest $\mathrm{X}$-ray was negative. She had been placed on isoniazid for treatment of latent TB infection (LTBI) approximately 2 months before she developed neck pain. Her infecting strain of MTB showed an identical genotype to the isolates from Cases 1 and 2.

Finally, in May 2000, an 81-year-old man (Case 5) was diagnosed with smear-negative, culture-positive pulmonary TB at a hospital in another part of the state. Genotype of his MTB isolate showed an exact match with the genotype of the three cases associated with this outbreak (see Figure 2.). After the genotypic match was discovered, questioning of the patient revealed that his wife had been a resident in Nursing Home A when Case 3 was there with a cavitary lung lesion. Case 5 frequently visited his wife there, and he had known Case 3. Case 5's wife remains well and TST-negative.

A search of the Arkansas genotype database, containing 1,514 isolates, for additional organisms matching the 15-band genotype associated with this outbreak revealed no additional exact match. Two isolates were found with restriction fragment length polymorphism patterns that differed from the 15-band genotype by one or two bands. These isolates were obtained from patients who died in 1995 and 1997. No epidemiological link could be found between these two patients and those in this outbreak.

\section{DISCUSSION}

In this outbreak, a 91-year-old woman developed TB after admission to a nursing home. Over a period of 8 months, she developed a cough with sputum production and became progressively weaker. She was hospitalized and treated 


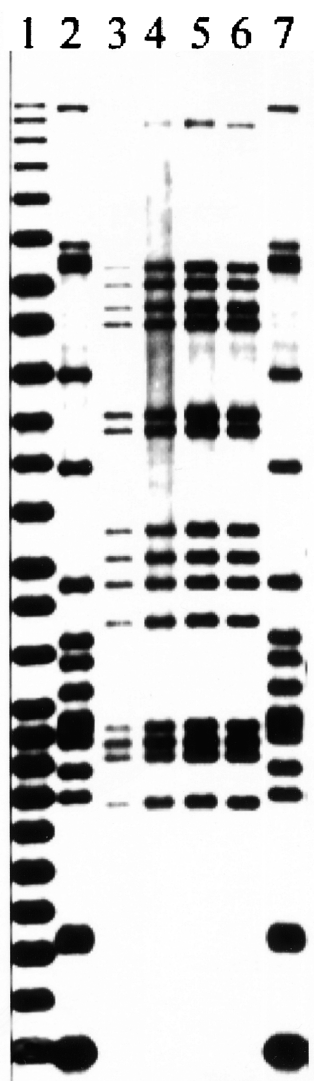

Figure 2. IS6110 restriction fragment length polymorphism patterns of deoxyribonucleic acid (DNA) extracted from Mycobacterium tuberculosis isolates from the four patients. Lane 1, molecular size standards that range in size from 0.70 to 15.0 kilobase pairs $(\mathrm{kbp})$. Lanes 2 and 7, DNA from laboratory strain H37Rv to which were added two molecular standards measuring $0.7 \mathrm{kbp}$ (bottom) and $13 \mathrm{kbp}$ (top). Lanes 3-6, DNA from case 2 (lane 3), case 4 (lane 4 ), case 1 (lane 5), and case 5 (lane 6).

for bacterial pneumonia without improvement and without ever having diagnostic studies for TB performed. A few weeks after her death, routine employee skin testing uncovered a TB outbreak that had been silently unfolding in two nursing homes, a community hospital, and the community. All together, four active cases of TB were found in addition to the source case, who died undiagnosed. She infected 52 employees, 23 residents and one visitor. The number of visitors is unknown; only 22 were evaluated, one developed active TB. We suspect that outbreaks of this type are not rare, but most go undetected. ${ }^{3-6}$ Greater attention to TB developing in nursing home residents is needed.

The quality of health care given in nursing homes has been criticized. On review of 62 consecutive deaths in a group of California nursing homes, 34 received "unacceptable care," and $30 \%$ of nursing homes in California have been cited for serious violations. ${ }^{9}$ Only a few nursing home deaths lead to autopsies. According to a 1990 report of nursing homes deaths in New York State, only $0.8 \%$ were autopsied. ${ }^{10}$

Approximately $5 \%$ of all reported TB cases are first diagnosed at death, and $60 \%$ of these are aged 65 and older. The odds of being diagnosed with TB at death is 11 times greater for persons aged 85 and older than for persons between the ages of 25 and 34. ${ }^{11}$ Apart from the harm brought to the patient, delayed diagnoses of TB also results in undetected transmission. One of the most common reasons for failure to diagnose $\mathrm{TB}$ in this setting is failure to consider TB in the differential diagnosis. ${ }^{12}$ Adequate sputum samples from geriatric patients are not easily obtained. Fiberoptic bronchoscopy may be required to obtain the necessary samples. Without bronchoscopy, up to $20 \%$ of geriatric TB patients can be missed. ${ }^{13}$ We wish to emphasize that TB should be considered in the differential diagnosis for every persistent pneumonia that fails to respond to antimicrobial treatment.

One of the largest reservoirs of LTBI in the United States and worldwide is the geriatric population, and the geriatric age group represents the fastest growing segment of the U.S. population. ${ }^{14}$ Older persons frequently have one or more conditions, including immunosuppression, diabetes mellitus, chronic renal failure, malignant neoplasms, weight loss, and residence in congregate settings, that place them at high risk for TB.

The American Thoracic Society and the Centers for Disease Control and Prevention published new recommendations for targeted testing and treatment of LTBI in $2000 .{ }^{15,16}$ They focus on identifying persons who are at high risk of developing TB if infected and recommend treatment of LTBI in such persons regardless of age. If these recommendations are widely followed, the number of older persons who progress from LTBI to active TB may decline significantly. This will be an important strategy for the elimination of TB in the United States, particularly as the number of persons aged 65 and older increases from 35 to 70 million over the next 30 years. Many of these persons will be living in congregate settings other than nursing homes, such as retirement communities and assisted living facilities, where screening for LTBI is not currently emphasized. In addition, this strategy will be important for reducing the incidence of TB in older foreignborn members of our society, who emigrated as young persons from countries with a high prevalence of $\mathrm{TB} .{ }^{17}$ Therefore, it makes sense to focus on the high-risk members of our geriatric population for targeted testing and treatment of LTBI if the elimination is to be embraced as a national goal.

\section{CONCLUSION}

A 91-year-old nursing home resident died of undiagnosed TB and transmitted infection to 80 persons. Four cultureproven TB cases resulted from this undetected case, including a nursing home resident, a nursing home employee, a hospital employee, and a visitor to the nursing home. The outbreak was discovered when routine annual screening of nursing home staff indicated that several staff members had been recently infected. Treatment for LTBI was offered to all the nursing home residents and healthcare workers who were found to have been recently infected, and the active cases were effectively treated, ending the outbreak.

\section{ACKNOWLEDGMENTS}

The authors acknowledge Dory Moers, Elisia White, Donnetta McGriff, and Billie Nott and many more public 
health nurses for their valuable time and contribution in helping investigate and control this outbreak. The authors specially thank Don Cunningham, Stewart Matthews, and Drs. John C. Shultz and William W. Stead for their valuable help, guidance, and input in preparation of this manuscript.

\section{REFERENCES}

1. Dutt AK, Stead WW. Tuberculosis. Clin Geriatr Med 1992;8:761-775.

2. Anonymous. Prevention and control of tuberculosis in facilities providing long-term care to the elderly: Recommendations of the Advisory Committee for Elimination of Tuberculosis. MMWR Morb Mortal Wkly Rep 1990;39: 7-20.

3. Stead WW, Lofgren JP, Warren E et al. Tuberculosis as an endemic and nosocomial infection among the elderly in nursing homes. N Engl J Med 1985;312:1483-1487.

4. Stead WW. Tuberculosis among elderly persons: An outbreak in a nursing home. Ann Intern Med 1981;94:606-610

5. Anonymous. Tuberculosis-North Dakota. MMWR Morb Mortal Wkly Rep 1979;27:523-525.

6. Munger R, Anderson K, Leahy R et al. Tuberculosis in a nursing care facility-Washington. MMWR Morb Mortal Wkly Rep 1983;32:121-128.

7. Strahan GW. An overview of nursing homes and their current residents: Data from the 1995 National Nursing Home Survey. Adv Data 1997;280:1-12.
8. Van Embden, Cave MD, Crawford JT et al. Strain identification of Mycobacterium tuberculosis by DNA fingerprinting: Recommendations for a standardized methodology. J Clin Microbiol 1993;31:406-409.

9. Anonymous. California Nursing Homes: Care Problems Persist Despite Federal and State Oversight (Publication no. HEHS-98-202). Washington, DC: U.S. General Accounting Office, 1998.

10. Katz PR, Seidel G. Nursing home autopsies. Survey of physicians and practice patterns. Arch Pathol Lab Med 1990;114:145-147.

11. Bobrowitz ID. Active tuberculosis undiagnosed until autopsy. Am J Med 1982;72:650-658.

12. Rieder HL, Kelly GD, Bloch AB et al. Tuberculosis diagnosed at death in the United States. Chest 1991;100:678-681.

13. Patel YR, Mehta JB, Harvill L et al. Flexible bronchoscopy as a diagnostic tool in the evaluation of pulmonary tuberculosis in an elderly population. $J$ Am Geriatr Soc 1993;41:629-632.

14. Hobbs BF, Damon BL. The sixty-five plus in the United States: United States Census Bureau. Current Population Report, Special Studies. Washington, DC: U.S. Government Printing Office, 1996. Available online: http://www.census.gov/prod/ 1/pop/p23-190/p23-190.pdf

15. Anonymous. Targeted tuberculin testing and treatment of latent tuberculosis infection. Am J Respir Crit Care Med 2000;161:S221-S247.

16. Cohn DL, O'Brien RJ, Geiter LJ et al. Targeted tuberculin testing and treatment of latent tuberculosis infection. MMWR Morb Mortal Wkly Rep 2000;49:1-51.

17. Zuber PL, McKenna MT, Binkin JN et al. Long-term risk of tuberculosis among foreign-born persons in the United States. JAMA 1997;278:304-307. 